\title{
Alles ist Zahl?
}

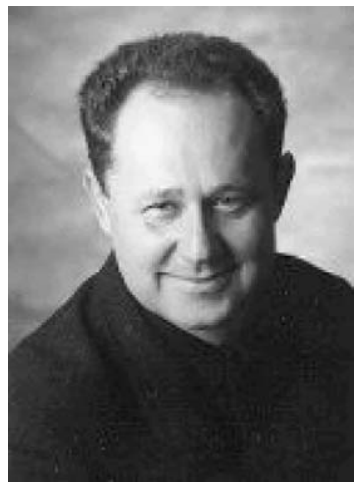

Prof. Dr. med. Manfred Wildner

\section{Bibliografie}

DOI http://dx.doi.org/ 10.1055/s-0033-1361126

Gesundheitswesen 2013;

75: 787-788

(c) Georg Thieme Verlag KG

Stuttgart · New York

ISSN 0941-3790

Korrespondenzadresse Prof. Dr. Manfred Wildner Bayerisches Landesamt für Gesundheit und Lebensmittelsicherheit

Veterinärstraße 2

85764 Oberschleißheim

manfred.wildner@|gl.bayern.de
Der Ausspruch „Alles ist Zahl“ wird dem griechischen Philosophen Pythagoras von Samos (570 v. Chr. bis 510 v. Chr.) zugeschrieben. Ob Pythagoras, ungeachtet der Nennung im Mathematikunterricht, tatsächlich zu den Pionieren der Mathematik gehörte, ist wissenschaftlich ungesichert. Kulturgeschichtlich ist er sicherlich bedeutsam. Die von ihm gegründete Schule legte Wert auf eine „pythagoräische“ Art des Lebens, d.h. eine disziplinierte, bescheidene - damit potentiell gesunde - und der Wahrheit verpflichtete Lebensweise. Ein Pendant in der Moderne wäre wohl am ehesten der „Lifestyle of Voluntary Simplicity“ (LOVOS), ein einfaches Leben als Gegenentwurf zu einer konsumorientierten Überflussgesellschaft, oder zumindest ein Lebensstil bewussten und gezielten Konsumierens („Lifestyle of Health and Sustainability“, LOHAS). Während das einfache Leben wohl ein Minderheitenphänomen geblieben ist, zumindest soweit es freiwilliger $\mathrm{Na}$ tur ist, hat sich die Bedeutung der Zahlen weltweit als „philosophischer Blockbuster“ erwiesen. Mathematik ist für viele Wissenschaftszweige geradezu das Merkmal und Echtheitssiegel ihrer Wissenschaftlichkeit. Sie hat nicht nur als Grundlage von (Bio-)Physik, Physiologie und (Bio-)Chemie Bedeutung, sondern auch in Form der Biometrie und Biostatistik im Bereich von klinischer Forschung und Public Health sowie allgemein in den empirischen Sozial-, Wissenschafts- und Lebenswissenschaften.

Konzeptionell hat unser moderner Umgang mit Zahlen Impulse und Entwicklungsschübe aus verschiedenen Epochen und Kulturräumen erhalten und ist auch in der Gegenwart nicht abgeschlossen. Die als „arabische“ Zahlzeichen gebräuchlichen Ziffern sind besser als arabisch-indische Ziffern zu bezeichnen. Sie entstammen der altindischen Brahmi-Schrift und deren 9 Zahlzeichen mit einem zusätzlichen als Kreis geschriebenem Zeichen für die Null. Dieses „Nichts“ als Stellzeichen im Dezimalsystem war eine Revolution. Die Bezeichnung „Ziffer“ ist in diesem Sinne auch abgeleitet von arabisch „as-sifr“ (Null, Nichts), der Übersetzung des Sanskrit-Bezeichnung „sunya“ für „leer“ [1].

Ihren zweiten großen und ebenfalls weltweiten Durchbruch haben die Zahlen im Binärsystem $(0,1)$ erlebt. Der Binärcode ist Grundlage der infomationellen Prozesse in Computern, auf Zahlenfolgen basierenden Rechnern, in Gestalt von Handheld Devices, Tabloids, Laptops, Desktops und Großrechenanlagen bis hin zu Cloud Computing, Internet und Cyberspace - wenn man so will Ausdruck einer vom Binärcode getragenen, weltumspannenden Sphäre zunehmend vernetz- ter geistiger Aktivitäten („Noosphäre“). In einer kulturellen Reflexion dieser technologischen Errungenschaften könnten damit alte philosophische Fragen aktualisiert gestellt werden: Sind wir, die wir nun selber virtuelle Welten erzeugen und in virtuellen Welten Identitäten annehmen können, vielleicht auch selber nur rechnerische Erzeugnisse eines gigantischen Computerprogramms, Produkte einer Zahlen-Matrix? Reicht unser Personensein vielleicht nur bis zu einem Dasein als „Avatar“ in der uns zugänglichen Welt? Spätestens an diesem Internet-Begriff, wiederum mit Herkunft aus dem Sanskrit (Avatara: „Herabkunft"), soll die Spekulation enden.

Die (All-)Gegenwart der Zahlen ist auch ohne derartige Spekulation und ganz im Konkreten gut zu fassen: Im Gesundheitswesen in Form von Leistungsstatistiken und Personalzahlen, als ökonomische Berechnungen und Planungsszenarien, als Laborwerte, Grenzwerte und Verlaufskontrollen und natürlich in Forschungszusammenhängen als elaborierte Berechnungen. In unserer modernen wissensbasierten und evidenzorientierten Welt sind Signifikanzschwellen, Evidenzgrade und Metaanalysen geradezu zu Eckpfeilern des praktischen Medizinbetriebes und damit auch des medizinischen Angebotes geworden. Sie erscheinen als Garanten und Koordinaten in einer zunehmend unübersichtlichen, komplexen Informationsgesellschaft. Wer die Zahlen kennt, hat einen Informationsvorsprung - zumindest, solange die Zahl und der zugehörige Kontext ausreichend verstanden sind. Schwierigkeiten bestehen naturgemäß im „Begreifen“ sehr großer und sehr kleiner Zahlen, die außerhalb unserer alltäglichen Lebenswirklichkeiten liegen. Ist uns - um hier nur ein Beispiel zu geben - im Verständnis der statistisch signifikanten Ergebnisse aus „großen“ Studien ausreichend bewusst, dass sich diese gerade bei hohen Teilnehmerzahlen auf sehr kleine Unterschiede beziehen können? Deren praktische Relevanz, auch bei gegebener Signifikanz, noch einmal gesondert zu bewerten ist? Ein möglicher Ansatz, sich in diesem bisweilen undurchdringlich erscheinenden Dschungel präsentierter Informationsvielfalt und Informationsunsicherheit nicht nur zu Recht zu finden, sondern auch Wege zu weisen, ist die Erarbeitung von wissenschaftlichen Leitlinien, mit ihren Qualitätsgraden S1-S4. Bedenklich ist dieser Ansatz dann, wenn sich derartige Leitlinien auf Metaanalysen bzw. Forschungsarbeiten abstützen, welche im Nachhinein als unzuverlässig eingestuft werden. Ein Beispiel dafür ist die S3-Leitlinie zur Behandlung der Psoriasis vulgaris wegen einer vermuteten möglichen Einflussnahme 
pharmazeutischer Unternehmen [2]. Ein weiteres Beispiel ist die Empfehlung zur Verwendung von Betablockern bei chirurgischen Risiko- bzw. Hochrisikoeingriffen. Hier hat eine Re-Analyse publizierter randomisierter kontrollierter Studien unter Ausschluss von mit hoher Wahrscheinlichkeit manipulierten Studienergebnissen ein beunruhigendes Ergebnis gezeigt [3]. Eine Re-Analyse allein auf Basis der Originalarbeiten mit verlässlichen Ergebnissen kam zu einer zu vielen Leitlinienempfehlungen divergenten Empfehlung hinsichtlich des Einsatzes von Betablockern in der oben genannten Patientengruppe. Diese Studie wirft die Frage auf, ob sich das Sterberisiko durch die nicht gerechtfertigte Vergabe von Betablockern um ein Viertel erhöht, was sich wiederum in Tausende von vermeidbaren operativen Todesfällen übersetzen ließe.

Damit stellt sich die Frage, inwieweit - gerade weil Zahlen allgegenwärtig scheinen - diese Zahlen auch zutreffen. Gilt womöglich das Gegenteil: alle Zahlen sind falsch? Lässt sich daraus eine Ablehnung von Metaanalysen und anderen zahlengestützten Instrumenten des Erkenntnisgewinns ableiten? Doch wohl eher nicht - das Problem ist wie so oft „menschliches Versagen“. Vielleicht auch eine in unserer menschlichen Natur begründete und dem Menschen somit eigentümliche, vorgegebene Unvollkommenheit, nicht ein Versagen der wissenschaftlichen Methode an sich. Metaanalysen haben auch zur Wendung medizinischer Praxisstile zum Besseren beigetragen, wie z.B. die Analysen zur Volumenersatztherapie mit Hydroxyethylstärke [4]. In der akademischen Diskussion wird allerdings auch die Hypothese vertreten, dass alle oder zumindest die meisten Ergebnisse empirisch-statistischer Forschung falsch sein könnten [5]. Mögliche Argumente für diese Ansicht begründen sich vor allem in der Kritik an einem von mancher Seite propagierten hypothesenfreien Vorgehen allein auf Basis von „big data“, wie dies bei genom-wide association studies (GWAS) mit buchstäblich zigtausenden, multiplen statistischen Testungen zum Einsatz kommen kann. Mögliche Argumente gegen diese Ansicht sind ebenfalls stichhaltig. Sie dürften im konventionellen wissenschaftlichempirischen Arbeiten mit seinem Iterieren zwischen Hypothesengenerierung unter Rückgriff auf verschiedene Erkenntnisquellen und anschließender empirischer, hypothesengesteuerter und fokussierter Prüfung auch tatsächlich „stechen“.

Schlechte Dinge gehen an sich selbst zugrunde, gute Dinge an ihren Übertreibungen. Möglicherweise ist ein deterministischer Ansatz, welcher gesundheitsbezogene Ergebnisse in der Individualmedizin wie auch in der Bevölkerungsmedizin auf Nachkommastellen genau festhalten und dingfest machen will, in der Medizin bzw. in den Lebenswissenschaften ähnlich verfehlt wie auf vielen Gebieten in der Physik, schon aufgrund der Warnungen, die uns die Chaostheorie bei kleinen Unschärfen der Ausgangsdaten in komplexen Systemen mit auf den Weg gibt. Einen gewissen Ausweg aus dem deterministischen Dilemma bietet ein probabilistisches Zahlenverständnis - also der Umgang mit Zahlen als Bezeichnung für Wahrscheinlichkeiten. Diese können durchaus Erkenntnisse befördern, auch wenn sie im Einzelfall keine absoluten Sicherheiten in die eine wie in die andere Rich- tung zulassen. Beispielsweise lässt ein positiver Quantiferontest in den meisten Fällen eine Infektion mit Tuberkuloseerregern vermuten, kann aber auch fälschlich positiv sein. Ebenso lässt sich die Aussage treffen, das eine Grippe- oder Masernimpfung wahrscheinlich vor der Erkrankung schützen wird, wobei eine $100 \%$-ige Serokonversion und ein damit verbundener 100\%-iger Schutz nicht zugesichert werden kann. Trotz dieser Unsicherheiten sind Labortests und Schutzimpfungen hilfreich und von hoher praktischer Relevanz, sowohl in der Individual- wie auch in der Bevölkerungsmedizin bis hin zur Ausrottung von Geiseln der Menschheit wie den Pocken.

In diesem angestrebten verständigen Sinn werden auch in dieser Ausgabe unserer Zeitschrift wieder Erkenntnisse aus den verschiedensten Bereich des Gesundheitswesen berichtet: $\mathrm{Zu} \mathrm{Ge-}$ sundheitsreformen und Versichertenpräferenzen, zu direkten Krankheitskosten von Diabetes mellitus in Deutschland, zum Zusammenhang von Deprivation im Wohnumfeld und Diabetes mellitus sowie zur Gesundheitskompetenz türkischstämmiger Menschen mit Diabetes, zu konservativer und chirurgischer Adipositas-Therapie, zum Einfluss nicht-klinischer Faktoren auf Innovationen bei Implantaten, zu psychologischen Interventionen bei Patienten mit chronischen Rückenschmerzen, zur schulärztlichen Bewertung des Förderbedarfs der kindlichen Sprachentwicklung, zum Weg aus dem Dickicht des Formulardschungels bei Anschlussrehabilitation bzw. Anschlussheilverfahren sowie zum gewünschten und tatsächlichen Sterbeort am Beispiel Rheinland-Pfalz.

Alles ist Zahl? Ein weiterer, aktuell gerade bei komplexen Interventionen und Sachverhalten viel diskutierter Weg verbesserten Erkenntnisgewinns ist eine methodische Erweiterung: die Integration von qualitativen und quantitativen Verfahren, sog. Mixed methods-Designs. Im Zusammenhang mit Individual- und Bevölkerungsgesundheit sei dazu ein Aphorismus berichtet, der dem Maler Ernst Fuchs (geboren 1930), einem Vertreter der Wiener Schule des fantastischen Realismus, zugeschrieben wird: „Gott zählt Menschen nicht 1, 2, 3, sondern 1, 1, 1.“ Diese komplementäre Zählweise - qualitative Forschung par excellence - dürfte auch in den modernen diesseitigen Gesundheits- und Lebenswissenschaften ihre Berechtigung haben.

\section{Literatur}

1 Ifrah G, von Plasen A. Universalgeschichte der Zahlen. Frankfurt: Verlag Zweitausendeins; 2008

2 Schott G, Dünnweber C, Mühlbauer B et al. Besteht ein Einfluss pharmazeutischer Unternehmen auf Leitlinien? Deutsches Ärzteblatt 2013; 110: $575-583$

3 Buri S, Shun-Shin MJ, Cole GD et al. Meta-Analysis of secure randomised controlled trials of Beta-blockade to prevent perioperative death in non-cardiac surgery. Heart, Published Online First [11.09.2013] doi:10.1136/heartjnl-2013-304262

4 Hartog CS, Welte T, Schlattmann P et al. Fluid replacement with hydroxyethyl starch in critical care - a reassessment. Dtsch Arztebl Int 2013; 110: 443-450 doi:10.3238/arztebl.2013.0443

5 Ioannidis JPA. Why most published research findings are false. PLoS Medicine 2005; 2: e124URL http://www.plosmedicine.org/article/ info:doi/10.1371/journal.pmed.0020124 